Annuaire suisse de politique de développement

\title{
Les partenariats public-privé, enjeux et défis pour la coopération au développement
}

Catherine Schümperli Younossian et Edouard Dommen

\section{(2) OpenEdition}

1 Journals

Édition électronique

URL : http://journals.openedition.org/aspd/335

DOI : 10.4000/aspd.335

ISSN : 1663-9669

Éditeur

Institut de hautes études internationales et du développement

Édition imprimée

Date de publication : 1 octobre 2005

Pagination : 9-19

ISSN : 1660-5934

\section{Référence électronique}

Catherine Schümperli Younossian et Edouard Dommen, « Les partenariats public-privé, enjeux et défis pour la coopération au développement », Annuaire suisse de politique de développement [En ligne], 24-2 I 2005, mis en ligne le 18 février 2010, consulté le 21 septembre 2020. URL : http://

journals.openedition.org/aspd/335; DOI : https://doi.org/10.4000/aspd.335 


\title{
Les partenariats public-privé, enjeux et défis pour la coopération au développement
}

\author{
Catherine Schümperli Younossian* \\ en collaboration avec Edouard Dommen**
}

\begin{abstract}
A
border la question des partenariats public-privé (PPP) dans la coopération au développement s'est avéré une tâche périlleuse et délicate, tant la notion est difficile à cerner du fait de ses acceptions diverses, et laisse apparaître des positions tranchées, voire émotionnelles. Non seulement il n'existe pas de définition reconnue de la notion de PPP qui puisse servir de référence commune aux différents acteurs de la coopération au développement, mais cette notion est en outre imprécise en ce qu'elle se réfère à plusieurs niveaux analytiques.
\end{abstract}

\section{Les PPP en quête d'une définition...}

Le premier niveau concerne le nouveau paradigme de développement qui vise à favoriser des partenariats avec le secteur privé du monde de l'économie, créant ainsi un cadre de référence innovant. Trois éléments majeurs ont permis à cette nouvelle orientation de s'imposer. Au début des années 1990, la Banque mondiale fut la première institution multilatérale à préconiser les PPP, notamment dans le domaine des infrastructures. Cette option a été rapidement relayée par les agences bilatérales de développement participant à la mise en œuvre du «cadre intégré de développement». En 1999, le secrétaire général de l'ONU, Kofi Annan, lance, à l'occasion du Forum économique de Davos, un «Pacte mondial», le Global Compact, qui cherche à stimuler l'application, par les entreprises transnationales, des droits sociaux et économiques ainsi que des normes environnementales. Enfin, lors de la Conférence de Monterrey sur le financement du développement, en 2002, les gouvernements soulignent l'importance des PPP comme instrument permettant de mobiliser de nouvelles ressources pour permettre de se rapprocher des Objectifs du millénaire. Dans un contexte de stagnation, voire de baisse de l'aide publique au développement, la création de nouveaux partenariats s'impose.

Un deuxième niveau s'intéresse aux diverses relations nouées entre les organisations internationales et les entreprises privées. L'étiquette de PPP va ainsi être appliquée à des initiatives aussi différentes que le Pacte mondial, des activités de sponsoring ou des projets conjoints. Afin d'illustrer ce niveau, l'encadré 1 cidessous présente deux exemples de partenariat public-privé multilatéral (PPPM). Cependant, le présent ouvrage ne se concentre pas sur ce type de PPP.

* Chargée de recherche et d'enseignement à l'IUED, responsable du dossier.

** Président du Comité scientifique du RUIG (Réseau universitaire international de Genève). Edouard Dommen a rédigé les encadrés. 


\section{Encadré 1}

\section{Deux exemples de partenariat public-privé multilatéral (PPPM)}

Global Alliance for Vaccines and Immunization (GAVI)

Alliance mondiale pour les vaccins et l'immunisation

Le GAVI classe ses partenaires dans les catégories suivantes:

- gouvernements de pays industrialisés;

- gouvernements de pays en développement;

- ONG;

- organisations internationales (nommément l'OMS, I'UNICEF et le groupe de la Banque mondiale);

- une fondation: la Fondation Bill et Melinda Gates;

- instituts de recherche et de santé publique;

- industrie des vaccins de pays industrialisés (les entreprises sont regroupées au sein de la Fédération internationale de l'industrie du médicament FIIM);

- industrie des vaccins de pays en développement (il s'agit d'une association professionnelle ad hoc).

Site Internet du GAVI: <www.vaccinealliance.org >.

Global Public-Private Partnership for Handwashing with Soap

Partenariat public-privé mondial pour le lavage des mains au savon

Ce PPP, domicilié à la Banque mondiale à Washington, compte comme membres

- des organisations internationales: Banque mondiale, UNICEF;

- des agences de l'administration fédérale des Etats-Unis;

- plusieurs des plus importantes entreprises mondiales productrices de savon: Colgate-Palmolive, Procter and Gamble, Unilever, Hindustan Lever;

- une ONG états-unienne;

- une institution académique, la London School of Hygiene and Tropical Medicine.

Site Internet du partenariat: <www.globalhandwashing.org $>$.

Enfin, une nouvelle répartition des rôles entre le public et le privé dans la gestion de certaines tâches de développement, qui précédemment étaient du ressort de l'Etat, concerne la troisième approche possible des PPP.

Afin de nous permettre de clarifier cette notion et d'appréhender les PPP de manière ordonnée, nous nous sommes posé trois questions qui ont constitué le fil rouge de cet ouvrage et qui ont également guidé nos auteurs dans leurs réflexions:

๑ Comment cette nouvelle pratique a-t-elle émergé dans la coopération internationale?

- Quels sont les potentiels, mais aussi les risques et les limites, des PPP?

$\checkmark$ Comment créer des conditions-cadres qui permettent aux PPP de déployer des effets positifs tout en en limitant les effets inopportuns?

L'ouvrage, qui s'intéresse principalement au premier et au troisième niveau évoqués ci-dessus, a l'ambition de susciter un débat qui permette aux milieux intéressés d'approfondir et de clarifier les concepts utilisés, mais également d'expliciter les attentes des uns et des autres par rapport aux PPP. Le comité de l'Annuaire suisse de politique de développement nourrit l'espoir que le dossier contribue à «désidéologiser» un débat qui a été parfois trop fortement influencé par des idées préconçues sur les vertus et les faiblesses du secteur privé et du service public... 


\section{Encadré 2}

\section{Le "partenariat public-privé »: un mot déclic}

Le PPP relève du mot déclic. Ces mots ne sont pas censés renvoyer à un concept analytique; le leur reprocher, c'est se méprendre sur leur fonction, qui est d'éveiller une réaction et d'orienter une attitude. Ils sont censés rassembler et mobiliser. On peut leur appliquer les propos de Serge Latouche sur l'oxymore: le "procédé inventé par les poètes pour exprimer l'inexprimable est de plus en plus utilisé par les technocrates pour faire croire à l'impossible »a. Les associations émotionnelles et culturelles des mots déclics sont ainsi plus importantes que leur contenu sémantique.

Ces mots doivent d'abord attirer et rassembler. Quant à l'attirance, il faut se servir de mots aux associations émotives qui inspirent la sympathie, comme "partenariat" en l'occurrence. Quant à rassembler, les mots choisis doivent être - pour prendre une analogie médicale - à large spectre. L'idéal est d'évoquer tout et son contraire: personne ne peut s'en sentir étranger si le mot n'exclut rien.

Les mots déclics sont assez nombreux pour que l'on puisse dresser une description du cycle de vie de la catégorie en général. D'entrée de jeu, le mot saisit une aspiration du moment en l'enrichissant d'associations et de sous-entendus émotionnels favorables. Afin d'élargir le cercle des partisans du mot, ses adeptes insistent d'une part sur ses résonances, s'efforçant de mieux exploiter le côté affectif du mot en y fourrant toutes sortes d'idées désirables. Ainsi, le mot "partenariat" sous-entend que les participants jouissent de respect mutuel et d'une certaine égalité entre eux, qu'ils œuvrent de concert pour atteindre un même objectif partagé.

D'autre part, les adeptes du slogan s'efforcent d'évacuer du mot tout élément de contenu analytique qui risque de susciter des oppositions. En effet, toute description d'un état de fait peut diviser les auditeurs entre le camp de ceux qui l'approuvent et ceux qui, la jugeant incorrecte, s'y opposent.

Entre-temps, des spécialistes ressentent le besoin de donner un sens technique précis au mot déclic pour en faire un outil de travail. Ils se mettent donc à l'œuvre pour lui définir un contenu analytique. Insistons que le processus qui s'efforce d'opérationnaliser le concept se déploie en même temps que celui qui œuvre à le vider de tout sens précis; les deux sont simultanés.

Or, plus on dompte le concept de la sorte, moins il titille l'imagination et plus il perd de son attrait émotionnel. En outre, toute définition opérationnelle va s'attirer des inimitiés de la part d'adeptes d'autres façons d'appliquer le concept ou d'autres structures d'analyse. Plus on précise le contenu du mot, moins il compte d'amis.

Bref, pour un mot déclic, de deux morts l'une: soit la dynamique émotionnelle prend le dessus et le mot en devient tellement vide de sens qu'il meurt d'inanition; soit le contenu technique l'emporte et à force de se préciser il se fait tellement d'ennemis qu'on l'abat. Le cycle de vie du mot "partenariat publicprivé " semble s'annoncer court: répandu à partir du début des années 1990, le mot paraît déjà s'approcher de sa phase terminale.

a Serge Latouche, "Le développement peut-il être "durable" ?", L'Ecologiste, n 6, hiver 2001, pp. 38-39.

Edouard Dommen 
L'ouvrage comporte quatre parties. La première, "Genèse et contour d'un concept», présente les conditions et les raisons de l'émergence de cette notion dans la coopération au développement et offre aux lecteurs certains éléments qui permettent d'en esquisser les contours. La notion de partenariat public-privé est entrée dans le vocabulaire de la coopération au développement depuis le début des années 1990. Mais peut-on vraiment affirmer que la collaboration entre le secteur privé et le secteur public soit nouvelle? Ces interactions existent depuis toujours dans des formes diverses et variées. L'innovation réside dans le fait qu'elles s'inscrivent dans un «nouveau paradigme politique» visant précisément à encourager ce type de partenariat. Frédéric Lapeyre revient sur les grandes étapes de l'évolution de la coopération au développement et analyse les dispositifs récemment mis en œuvre, tels le «cadre intégré de développement» et les «stratégies de réduction de la pauvreté» prônés par les institutions financières internationales. Selon l'auteur, ces nouveaux dispositifs «matérialisent le nouveau paradigme du développement qui a profondément bouleversé la distribution des rôles et des responsabilités des acteurs étatiques et non étatiques». L'article tend à montrer que la mise en œuvre des PPP implique une gestion de conflits d'intérêts entre différentes parties prenantes, ce qui nécessite dialogue et négociation pour pouvoir les surmonter et parvenir à un compromis. Et de souligner la nécessité de reconnaître la participation de la société civile, notamment le rôle des populations et de leurs organisations représentatives, pour permettre la réalisation de projets de développement viables.

Par le terme même de «partenariat», on admet l'existence de deux acteurs au moins, certes distincts mais de même niveau, complémentaires dans leurs attributions, et pouvant utilement collaborer sur un objectif commun (qui reste à définir). Partant de ce constat théorique, Peter Ulrich et Florian Wettstein mettent en lumière les systèmes de motivations des protagonistes impliqués dans des PPP dans le domaine de la politique de développement. Et les auteurs de présenter les perspectives d'éthique de développement que devraient endosser les entreprises, c'est-à-dire une prise en charge de responsabilités sociales et publiques, élément intégral de leur effort légitime de maximisation des bénéfices. En guise de démonstration, l'article se conclut sur une analyse critique du Pacte mondial mis en œuvre sous les auspices des Nations unies.

Si l'article précédent interroge les principes fondamentaux qui régissent les sphères du public et du privé, les différents constituants d'un PPP vont notablement influencer la nature, voire l'esprit des partenariats conclus, selon les acteurs concernés. La «taxinomie» présentée dans l'encadré 3 par Edouard Dommen s'avère un instrument utile pour affiner l'analyse des motivations des partenaires potentiels. De plus, afin d'orienter le lecteur au travers des contributions du dossier, nous avons identifié, par le nom de leurs auteurs, les articles qui abordent de manière plus approfondie le rôle ou les activités des constituants mentionnés. 


\section{Encadré 3}

\section{Les différents constituants d'un partenariat public-privé}

Pour être considéré comme public-privé, un partenariat doit compter au minimum deux partenaires, dont l'un de la catégorie des organisations officielles (secteur public) et l'autre du secteur des entreprises privées ou de la société civile.

\section{Secteur public}

- International: comprend notamment les organisations onusiennes (OMS, UNICEF, Organisation des Nations unies pour l'éducation, la science et la culture [UNESCO]...) ou les institutions de Bretton Woods et les banques de développement.

[1] Dossier PPP: F. Lapeyre, P. Ulrich/ F. Wettstein, J. Richter.

- National: comprend l'Etat dans ses composantes civile et militaire, et éventuellement les entreprises d'Etat.

[1] Dossier PPP: B. Chassot Gétaz/K. von Däniken/T. Zeller (seco/DDC), W. Gruber/D. Rothenberger (seco), S. Cohen (Etat de Genève), X. Tschumi Canosa, G. Carbonnier.

- Local: comprend les différents niveaux d'autorité publique à l'intérieur de l'Etat (provinces, municipalités...), et éventuellement les entreprises qui leur appartiennent.

[ㅁ] Dossier PPP: M. Tidjani Alou.

\section{Secteur des entreprises privées}

- Les entreprises:

- les sociétés transnationales, qui peuvent former un partenariat au niveau de l'organe central, d'une filiale nationale ou d'un établissement (p. ex. une usine ou une succursale) dans un lieu donné:

- les grandes sociétés nationales;

- les PME: étrangères ou du pays.

[1] Dossier PPP: S. Koch.

- Les associations professionnelles: internationales, nationales ou locales, elles comprennent des organisations recouvrant plusieurs branches (les chambres de commerce p. ex.) ou un secteur particulier (un office du tourisme), ou encore des associations réunissant plusieurs entreprises d'une même branche (p. ex. la Fédération internationale de l'industrie du médicament FIIM).

\section{[ㅁ] Dossier PPP: S. Cohen.}

- Les institutions philanthropiques d'entreprise: plusieurs entreprises entretiennent diverses fondations d'entreprise (p. ex. "Novartis Foundation for Sustainable Development») ou d'autres structures philanthropiques (p. ex. Nestlé, voir <www.community.nestle.com>).

\section{Société civile}

- Les ONG participent de plain-pied à de nombreux PPP.

- Les communautés comprennent des entités de la société civile qui ne sont pas des autorités publiques (p. ex. des associations de quartier).

[1] Dossier PPP: S. Hoffmann, C. Eberlein.

- Les institutions philanthropiques autres que les fondations d'entreprise couvrent évidemment un spectre étendu. La Fondation Bill et Melinda Gates en constitue un exemple actif, notamment dans des PPPM de la santé comme le Fonds mondial de lutte contre le sida, la tuberculose et le paludisme. La fondation d'entreprise Fundación Aguas Argentinas, émanation d'un PPP, travaille avec des institutions publiques et avec des organisations communautaires.

\section{Institutions académiques ou de recherche}


Dans la deuxième partie de l'ouvrage, «Les partenariats public-privé dans la coopération au développement», les partenariats public-privé sont présentés en tant qu'instrument de la coopération au développement. Dans leur dimension opérationnelle, les PPP sont perçus comme une alliance entre entités publiques et privées qui s'associent en vue de remplir un objectif collectif. Ils sont supposés permettre l'amélioration de la gestion d'un projet d'intérêt public, sous la responsabilité mutuelle d'un acteur public et d'un opérateur privé. Le financement est également assuré par des ressources conjointes (privées et publiques) et formalisé par un contrat. Dans certains cas, les PPP sont devenus l'instrument d'une politique de privatisation promue par les institutions internationales; dans d'autres situations, ils permettent l'association de partenaires complémentaires pour la réalisation de projets de développement. Comme mentionné précédemment, cette nouvelle approche est apparue dans un contexte de stagnation, voire de diminution, de l'aide publique au développement. C'était une manière de trouver de nouvelles sources de financement.

La coopération suisse, par les activités tant de la Direction du développement et de la coopération (DDC) que du Secrétariat d'Etat à l'économie (seco), participe à des projets de PPP et les soutient. Dans un premier article conjoint de la DDC et du seco, Brigitte Chassot Gétaz, Kathi von Däniken et Thomas Zeller plaident pour un renforcement de la collaboration entre les pouvoirs publics et l'économie privée qui aurait «pour avantage essentiel d'engendrer un effet multiplicateur et une croissance économique à long terme». Si les deux principaux organismes suisses de coopération poursuivent, par ce biais, le même objectif (à savoir atténuer la pauvreté et contribuer à la réalisation des Objectifs du millénaire), les modalités et les lignes directrices régissant leur collaboration avec le secteur privé leur sont propres. En matière de PPP, le seco s'est spécialisé dans le domaine des infrastructures. Une présentation détaillée de Werner Gruber et Dieter Rothenberger permet de cerner les objectifs et les instruments du seco, illustrés par des exemples, tels l'approvisionnement en électricité au Tadjikistan ou les PPP dans le domaine de l'eau en Tanzanie et en Bolivie.

Afin d'élargir la perspective, Xavier Tschumi Canosa présente les pratiques des coopérations allemande et française, et souligne l'importance grandissante des projets conduits selon ces modalités. L'auteur relève l'intérêt que recèlent les PPP pour lever des fonds supplémentaires devant servir à atteindre les Objectifs du millénaire, mais insiste sur l'importance d'un cadre institutionnel fort dans les pays en développement afin d'éviter les abus possibles de la part des partenaires impliqués. De même, il insiste sur la primauté de l'objectif de développement, dont les agences de coopération doivent être garantes.

Enfin, la parole est donnée à Sylvie Cohen (directrice des Affaires extérieures de l'Etat de Genève), qui présente la coopération au développement de son canton. Celui-ci s'est récemment doté des outils nécessaires pour soutenir des projets de développement proposés par des entreprises privées genevoises. 
Dans la troisième partie, «Enjeux et défis. Points de vue de différents acteurs», la parole est donnée à des représentants d'ONG, du secteur privé et des milieux académiques. Une attention particulière a été portée aux PPP dans le secteur de l'eau: plus de la moitié des articles du dossier abordent en effet le sujet des PPP dans ce domaine, en offrant différents éclairages et de nombreux exemples ${ }^{1}$. Ils font ainsi écho aux propos de Frédéric Lapeyre, qui relève, dans le premier article de l'ouvrage, que l'on compte aujourd'hui plus de 2300 PPP dans le secteur de l'eau et de l'assainissement, alors qu'ils étaient quasiment inexistants au début des années 1990. L'auteur constate que la promotion des partenariats et la redéfinition des champs de responsabilité public-privé ne sont toutefois pas sans poser de problèmes car, sous le couvert d'améliorer la qualité et l'accès aux services essentiels, ces politiques se sont traduites par des résultats parfois controversés lorsqu'ils ont été associés à la privatisation de ces services.

Christine Eberlein présente une approche critique des PPP, s'interrogeant sur les possibilités d'accès aux infrastructures pour les populations vivant au-dessous du seuil de pauvreté. Son point de vue reflète la position des ONG qui s'interrogent sur les conditions à remplir pour que les PPP puissent répondre aux problèmes de développement qu'ils sont censés résoudre. Elle relève les contradictions inhérentes au concept même et met en question les capacités de surmonter les tensions potentielles entre les intérêts des entreprises privées et les tâches incombant aux institutions publiques. Ainsi l'auteure, exemples à l'appui (construction de logements sociaux en Afrique du Sud, barrage en Ouganda, alimentation et assainissement de l'eau à El Alto, en Bolivie), montre les limites des PPP dans le domaine des infrastructures, notamment en ce qui concerne leur accès pour les plus pauvres. Selon elle, le «modèle des PPP ne peut réussir qu'abordé comme un processus ouvert, tenant compte de l'intérêt de tous les acteurs ». Elle étaie son propos en présentant l'exemple bolivien d'une coopérative qui assure l'alimentation en eau de la ville de Santa Cruz.

La privatisation du secteur public dans le domaine de l'eau est présentée à l'aide du cas du Niger. Sous la plume de Mahaman Tidjani Alou, le rôle de l'Etat en matière de gestion de l'eau est analysé, ainsi que l'avènement de nouveaux acteurs non étatiques. Pour l'auteur, la réforme du secteur de l'eau traduit d'abord la sanction d'une gestion publique défaillante et la nécessité de définir un nouveau cadre d'intervention de l'Etat, qui laisse apparaître de nouveaux acteurs non gouvernementaux, telles des entreprises privées ou des associations. Une analyse différenciée, entre l'hydraulique urbaine et l'hydraulique villageoise, permettra d'expliciter une logique multiforme, aux modalités d'application diverses selon une réalité socio-économique bien différente entre ville et campagne.

L'article de Sabine Hoffmann aborde la notion de partenariat par le biais des régimes institutionnels de propriété et de possession. Aux yeux de l'auteure, les partenariats posent inéluctablement des conditions institutionnelles qui définissent les droits, les obligations, les privilèges et les non-droits des acteurs concer-

I Il s'agit des articles de Frédéric Lapeyre; Werner Gruber et Dieter Rothenberger; Xavier Tschumi Canosa; Christine Eberlein; Mahaman Tidjani Alou; Sabine Hoffmann. 
nés. L'article expose les différentes logiques auxquelles sont soumis les acteurs dans le cadre de partenariats: rationalité économique (liée à une économie de propriété) ou raison écosociale (liée à une économie de possession). Quelle logique guide les acteurs? La réponse apportée par l'auteure s'appuie sur une étude de cas menée à Cochabamba, en Bolivie, concernant l'accès à l'eau potable.

Gilles Carbonnier appréhende la problématique des PPP sous l'angle de la privatisation des services publics dans les conflits armés. Sont ainsi abordées, d'un point de vue juridique et humanitaire, l'émergence des compagnies militaires privées, mais également la privatisation et la sous-traitance des services liés à l'approvisionnement en eau, à la gestion des lieux de détention et aux soins de santé. Trois questions majeures sont posées: Quelle est la compatibilité entre un objectif de maximisation du profit, propre aux entreprises privées, et celui du maintien de la sécurité et de la santé publique, propre aux Etats ? Comment exiger le respect du droit international par les sociétés privées et quelle est la responsabilité de l'Etat lorsque les activités de ces sociétés enfreignent le droit international humanitaire? Existe-t-il une réelle rationalité économique lors de privatisations, principal argument des responsables politiques qui les encouragent? Les réponses à ces diverses questions dépendront de la compréhension que les organisations humanitaires pourront développer de leurs relations complexes avec les opérateurs privés.

Stefanie Koch présente les motivations et les pratiques d'une entreprise privée, la firme Holcim, l'un des principaux fournisseurs mondiaux de ciment et de béton. L'auteure relève que les partenariats impliquant divers acteurs sont «multisectoriels» et doivent donc intégrer des motivations diverses, voire contradictoires. Elle distingue, pour le secteur privé, les partenariats stratégiques des partenariats opérationnels. Dans le premier cas, on se réfère aux organisations avec lesquelles une entreprise souhaite établir une relation de long terme, telles des agences de coopération ou des organisations non gouvernementales. C'est une première approche, la "phase exploratoire», qui permet d'identifier les partenaires possibles, de cerner les motivations des uns et des autres, de définir si les objectifs poursuivis sont compatibles et si la collaboration pourra se concrétiser dans l'élaboration d'un projet. Dans le cas des partenariats opérationnels, la première phase a été remplie avec succès, le partenaire identifié et les objectifs définis, et l'on entre dans la phase opérationnelle du projet commun. Une série d'exemples de PPP conclus entre la firme Holcim et différents partenaires illustrent le propos. Si l'auteure relève les nombreux avantages potentiels que recèlent les PPP, elle n'en demeure pas moins prudente quant aux risques de dérapage qui peuvent exister si le partenaire n'est pas adéquat.

\section{Points d'appui}

Enfin, la quatrième partie de l'ouvrage, «Points d'appui», contient une bibliographie riche d'une vingtaine de documents, ouvrages ou articles de référence, présentés de manière détaillée et critique. Judith Richter a accordé une attention particulière à des sources documentaires issues des organisations internationales, notamment des Nations unies, de la Banque mondiale et du Fonds monétaire international, qui définissent le nouveau paradigme de la coopération au 
développement visant à promouvoir une plus grande participation du secteur privé. Outre ces rapports, Judith Richter recense également les principaux auteurs ayant publié des ouvrages critiques par rapport aux nouvelles orientations des organisations internationales. Les lecteurs seront ainsi orientés vers des sources documentaires complémentaires, qui traitent une dimension de PPP peu abordée par les articles de ce dossier.

\section{Mise en perspective}

Avant de conclure cette introduction, et que le lecteur prenne connaissance des multiples facettes que recèlent les PPP, nous relevons l'intérêt qu'il y a à élargir le nombre d'acteurs impliqués dans la coopération au développement. Le développement est en effet une des tâches des secteurs tant public que privé. Tous doivent être engagés dans la lutte pur éradiquer la pauvreté, conformément aux Objectifs du millénaire. Toutefois, les nouvelles alliances que constituent les PPP doivent obéir à toute une série de conditions si elles veulent contribuer à la réalisation de ces objectifs.

Un des questionnements qui a guidé notre réflexion lors de l'élaboration de ce dossier concerne les conditions qui permettraient aux PPP de déployer des effets positifs tout en limitant les conséquences inopportunes. S'il est difficile de dresser un panorama précis des mesures à envisager, les réponses apportées par les différents auteurs au fil des contributions nous donnent toutefois un certain nombre de pistes, succinctement évoquées ci-dessous. La première concerne les conditions à remplir pour articuler les intérêts privés et publics en faveur du développement; la seconde s'intéresse aux lignes directrices définies par les partenaires eux-mêmes pour clarifier leurs objectifs; la troisième évoque la nécessité de créer un cadre juridique contraignant pour les différents acteurs; la quatrième accorde une attention particulière aux acteurs bénéficiaires des services susceptibles de faire l'objet d'un PPP; la cinquième piste, enfin, s'intéresse aux organes de régulation indispensables lors de la mise en œuvre d'un PPP.

La question fondamentale de savoir dans quelle mesure les PPP peuvent vraiment constituer un modèle durable d'articulation entre intérêts privés et publics en faveur du développement renvoie aux motivations des acteurs. Comme le montre l'encadré 4, les motivations des partenaires divergent bien souvent. Se pose la question du rapport de force entre le secteur privé et le secteur public. Quand le secteur privé est représenté par des entreprises multinationales au pouvoir considérablement plus élevé que les Etats avec lesquels il noue des partenariats, la sauvegarde des intérêts publics restera un sujet de préoccupation majeure. Par contre, il est indéniable que sur le plan local, des partenariats entre petites entreprises et les pouvoirs publics peuvent s'avérer profitables pour autant que les autorités soient à même d'imposer un cadre contractuel clair en faveur d'objectifs d'intérêt général. 


\section{Encadré 4}

\section{Eventail des motivations des partenaires}

Quel est l'intérêt pour le secteur privé de constituer un PPP?

1. Vendre les biens et services qui constituent l'objectif premier de l'entité privée en question:

1.1. pour en tirer un revenu;

1.2. pour contribuer à la maximisation des bénéfices.

2. Elargir le marché.

3. Verrouiller le marché.

4. Influencer le cadre politique et juridique dans lequel l'entreprise privée opère.

5. Lustrer son image de marque.

Quel est l'intérêt pour le secteur public de constituer un PPP?

1. Se fournir à meilleur coût, dans la mesure où

1.1. le secteur privé peut être plus efficace, dans le sens que le marché est trop occasionnel ou trop insignifiant pour que l'autorité publique s'y investisse;

1.2. les entreprises privées subissent moins de contraintes dans des choix qui ont un coût budgétaire important (notamment en ce qui concerne les conditions d'emploi).

2. Echapper à d'autres contraintes politiques ou juridiques.

3. Stimuler l'innovation.

4. Mieux profiter des connaissances techniques spécialisées d'entreprises privées

5. Sortir certains éléments des comptes publics (p. ex. transférer des montants de la dette d'administrations publiques aux nouveaux PPP).

E. D.

Les auteurs du dossier relèvent que l'association du secteur privé aux objectifs de développement ne va pas de soi et soulève de nombreuses questions. Et chacun d'exposer des idées pour atténuer les tensions ou cadrer les conflits d'intérêts. L'ouvrage présente les différents garde-fous ou lignes directrices dont se dotent les acteurs, tant publics que privés. Ainsi, les agences de coopération de la Suisse, de la France, de l'Allemagne et, au niveau local, du canton de Genève, de même que l'entreprise privée Holcim, dont les expériences respectives sont abordées dans ce dossier, ont toutes défini un cadre de référence pour leurs pratiques dans le domaine des PPP. Cet exercice leur semble nécessaire, sinon indispensable, pour clarifier leurs objectifs, voire leurs motivations, et définir les résultats escomptés. Ces documents servent souvent de base à la discussion entre les différents partenaires amenés à collaborer sur un projet commun. Dans sa bibliographie commentée, Judith Richter accorde une attention particulière aux démarches effectuées par les Nations unies et certaines de ses agences spécialisées pour définir des directives concernant la coopération entre les organisations internationales et les entreprises privées, cela afin de préserver les intérêts publics dans le cadre de PPP.

Si des lignes directrices, unilatérales et volontaires, semblent indispensables pour clarifier les objectifs des acteurs concernés, la création d'un cadre juridique contraignant pour réguler les activités des entreprises paraît nécessaire pour certains auteurs. Ainsi, Peter Ulrich et Florian Wettstein saluent la démarche de vouloir formaliser des liens entre le secteur privé et les Nations unies, notamment par le biais du Pacte mondial comme "plate-forme durable d'apprentissage et d'élaboration de normes de bonne conduite», mais s'interro- 
gent sur la crédibilité de l'exercice et suggèrent la mise en place de mécanismes permettant de vérifier et de faire respecter les dix principes du Pacte. Les auteurs n'hésitent pas à franchir un pas supplémentaire en affirmant que si les entreprises, parties prenantes du Pacte, voulaient vraiment faire preuve de «civisme», elles devraient majoritairement souscrire au projet de «Normes sur la responsabilité en matière de droits de l'homme des sociétés transnationales et autres entreprises », actuellement en discussion à la Commission des droits de l'homme.

Nombreux sont les auteurs qui rappellent que la notion de partenariat publicprivé ne concerne pas seulement les relations avec les entreprises, mais que les nouveaux partenariats doivent également tenir compte de la participation populaire à ce processus. Si Frédéric Lapeyre pose le cadre général dans lequel la participation populaire doit s'inscrire, de nombreux auteurs, s'appuyant sur des exemples concrets, corroborent cette nécessité (Christine Eberlein, Mahaman Tidjani Alou, Sabine Hoffman, Gilles Carbonnier). Ainsi est posée la question du rôle de la société civile, des usagers des services susceptibles de faire l'objet de PPP. Dans une dynamique de développement visant à sortir les populations les plus faibles de la pauvreté, il est essentiel que les concernés aient leur mot à dire. Ce sont eux qui disposent des solutions les plus adéquates. Une telle participation nécessite toutefois elle aussi des règles précises. Cette piste suggère que les PPP, pour qu'ils deviennent utiles au développement, soient conçus non comme un partenariat entre deux acteurs, Etats et entreprises, mais comme un partenariat entre trois acteurs, incluant, à côté des deux premiers, les usagers.

Force est de constater que de nouvelles alliances, comme celles des PPP, complexifient indéniablement les pratiques de la coopération au développement. Les contributions du dossier l'ont démontré, la mise en œuvre de projets impliquant des partenaires dont les motivations ou les intérêts divergent nécessite des dialogues et des régulations. Des organes de régulation, dont les formes et les pratiques varient d'un PPP à l'autre, apparaissent comme indispensables notamment pour concilier des intérêts souvent divergents et défendus par des acteurs fort hétérogènes. Cette régulation doit permettre de contribuer à améliorer les perspectives de développement dans un intérêt collectif. L'article de Mahaman Tidjani Alou offre à cet égard une illustration éclairante des difficultés qui peuvent surgir dans ce type d'exercice.

En publiant cet ouvrage, notre volonté est de clarifier un concept nouveau et de présenter les potentialités, mais également les limites, d'un instrument de développement auquel les agences de coopération ont de plus en plus recours. Les différentes contributions au dossier laissent apparaître des pistes de réflexion, dont chacune nécessite un approfondissement complémentaire. Mais nous espérons que le débat ainsi lancé sera poursuivi et permettra de renforcer le cadre institutionnel des partenariats public-privé afin que ceux-ci puissent déployer des effets positifs pour les plus nécessiteux de notre monde. 